\title{
IMMUNOLOGY OF MULTIPLE SCLEROSIS
}

\author{
Uleng Bahrun, Chelvi Wijaya \\ Department of Clinical Pathology, Faculty of Medicine, Hasanuddin University/Wahidin Sudirohusodo Hospital, Makassar, \\ Indonesia. E-mail: ulengbahrun68@gmail.com
}

\section{ABSTRACT}

Multiple Sclerosis (MS) is an autoimmune disease that causes myelin destruction in the Central Nervous System (CNS). Characteristics of this disease are perivascular infiltration by inflammatory cells, demyelination and loss of axons, accompanied by multiple plaque formation in the brain and spinal cord. According to the National Multiple Sclerosis Society, 400,000 people suffer from MS in the United States and about 2.5 million people worldwide. The disease is usually diagnosed in patients aged $20-45$ years and more often found in females than males with a ratio of 2: 1 . Nevertheless, the etiology of MS is still unknown, but it is suspected that genetic and environmental factors may induce a response to central nervous system autoantigen, such as Epstein-Barr Virus (EBV) infection. It then causes edema, demyelination, axon destruction and loss of oligodendrosites, resulting in neurological deficits, plaque formation, scarring and reduced brain volume. Multiple sclerosis symptoms and signs actually vary greatly depending on the location of the lesions and the course of the various diseases. Multiple sclerosis, moreover, can be divided into four clinical categories, namely Clinically Isolated Syndrome (CIS), Relapsing Remitting Multiple Sclerosis (RRMS), Secondary Progressive Multiple Sclerosis (SPMS) and Primary Progressive Multiple Sclerosis (PPMS). Diagnosis of Multiple sclerosis is established based on clinical symptoms and supporting investigations, such as MRI, CSF and neurological examination. In CSF examination, oligoclonal bands are found in over $90 \%$ of patients, considered as one of the laboratory criteria supporting the diagnosis of MS. There are four kinds of drugs that have been approved by the FDA as disease modifying therapy for the initial treatment of MS patients, namely interferon beta-1a, subcutaneous (SC) interferon beta-1a, interferon beta- $1 \mathrm{~b}$ and glatiramer acetate In conclusion, life expectancy in MS patients is only slightly reduced and survival is related to the disability occurred.

Key words: Multiple sclerosis, autoimmune

\section{INTRODUCTION}

A Parisian neurologist, Jean Martin Charcot was the first person contributing to the introduction of Multiple Sclerosis (MS) disease in 1868 by linking clinical features and pathological abnormalities of the patients. Charcot then revealed accumulation of inflammatory cells in the perivascular and demyelinating areas together with plaques found in the substrate of brain alba and spinal cord in patients with intermittent neurological deficits, resulting in the term "sclérose en plaques disseminées" or multiple sclerosis.1

Multiple sclerosis is an autoimmune disease that causes myelin destruction in the Central Nervous System (CNS). Characteristics of this disease are perivascular infiltration by inflammatory cells, demyelination and loss of axons, accompanied by mul- multiple plaque formation in the brain and spinal cord. $^{2,3}$

Myelin, moreover, is a protein-phospholipid material that encircles axons in a multilayer form. The myelin sheath of the central nervous system is composed of oligodendroglia cell membranes. Myelin serves to allow the axons to deliver impulses faster, therefore loss of myelin reduces impulse delivery speed and results in the manifestation of neurological dysfunction. The clinical manifestations of MS vary widely and have the characteristics of relapse and remission at the onset of the disease. ${ }^{4-6}$

\section{EPIDEMIOLOGY}

Multiple sclerosis is the most common cause of neurological disability in young adults. According to the National Multiple Sclerosis Society, 400,000 peo- 
ple suffer from multiple sclerosis in the United States and about 2.5 million people worldwide. The disease is usually diagnosed in patients aged $20-45$ years. Only $5 \%$ of patients under 10 years or above 50 years suffer from MS. Multiple sclerosis is also known to be more common in females than males with a ratio of $2: 1 .^{5-8}$

Furthermore, the incidence and prevalence of MS in the world varies widely. Multiple sclerosis is thought to be triggered by genetic and geographic factors. The highest prevalence of MS is in Northern and Central Europe, North America and Southern Australia. Asians and Africans have a lower prevalence compared with Caucasians. The prevalence of MS increases in proportion to the increase of the place distance from the equator, but there has been no satisfactory explanation of this phenomenon although some variables have been studied. ${ }^{5,7}$

\section{ETIOLOGY}

The etiology of MS is still unknown. Nevertheless, it is suspected that genetic and environmental factors are involved. A well-known theory argues that MS occurs in people with genetic susceptibility exposed to some environmental factors resulting in damage to the blood-brain barrier, the protective membrane, regulating the passage of the substance from the blood into the central nervous system. ${ }^{5,6,8}$ In addition, there is no specific gene that has been identified in MS. Studies on twins show an increased risk of $30 \%$ for obtaining MS in identical twins and $5 \%$ in non-identical twins. Several studies also have revealed that the Human Leukocyte Antigen (HLA) region on chromosome 6 corresponds to MS, namely HLA-DRB1 on chromosome 6p21. Meanwhile, nonHLA genes associated with MS are IL2RA and IL7R. ${ }^{5,9,10}$

Besides, environmental factors allegedly playing a role are viral infections, such as Epstein-Barr Virus (EBV) triggering mononucleosis to increase the risk of developing MS later in life. Studies show that MS patients tend to have higher antibody titers against EBV. In addition to infections, other environmental factors suspected to be associated with MS are less sun exposure and less vitamin D intake. ${ }^{5,9,10}$

\section{PATHOGENESIS}

Multiple Sclerosis is thought to occur as a result of a combination of genetic predisposition and exogenous factors from the environment that induce responses to autoantigen of central nervous system.
This process is believed to be initiated by autoreactive Th1 cells (not fully), activated peripherally by some unknown mechanisms, such as molecular mimicry with infectious agents, superantigens and others. These activated T cells then will proliferate, expressing some receptors and adhesion molecules as well as secreting proinflammatory mediators and metalloproteinases that activate the blood-brain barrier and interact with it to enter the brain.

After entering the brain, $T$ cells will be reactivated by a local autoantigen presented by $\mathrm{MHC}$ class II expressed by activated microglia, astrocytes and macrophages. This initiates local inflammatory reactions as well as secretion of cytokines, chemokines and other mediators by activated cells and other immune system components (macrophages, cytotoxic $T$ cells, B cells and complement). B cells play a role by forming immunoglobulins. Subsequent immunologic reactions to myelin, axon and glia cells are mediated by cytotoxic and cytokine cells, phagocytosis, proteases, antimyelin antibodies, complement, glutamate, as well as Nitric Oxide (NO). The result is edema, demyelination, axon destruction and loss of oligodendrosites that cause neurological deficits, plaque formation, scarring and reduced brain volume. ${ }^{2,11}$

There are several processes in the pathogenesis of MS (Figure 1), namely: ${ }^{11}$

1. Activation there are autoreactive CD4+ T lymphocytes in the circulation, activated by several processes, such as viruses, superantigens and others. These $T$ cells then can cross the blood-brain barrier.

2. Passing the blood brain barrier.

3. Presentation and stimulation of antigens In the central nervous system, antigen presenting cell, MHC class II and co-stimulatory signals (CD28, B-7.1) stimulate activation and proliferation of previously entered $T$ cells into Th1 and Th2 cells. Proinflammatory cytokines by Th1 cells (IL-2, IFN- $\gamma$, TNF- $\alpha$ ) then induce phagocytosis by macrophages and microglia, as well as inflammatory mediator (TNF- $\alpha, N O)$ and complement factor synthesis. Th2 cells secrete cytokines (IL-4, IL-5, IL-6) that activate $B$ cells to form antimyelin antibodies. All of them then cause damage to myelin. Th2 cells also produce IL-4 and IL-10 which suppress Th1 cells.

4. Demyelination 
The inflammatory process causes damage to the myelin and axons resulting in demyelination. Axons that should be wrapped by myelin become myelin loss resulting in a reduced impulse delivery rate and a neurological deficit.

5. Formation of scar tissue

When there is autoreactive T cell apoptosis, the inflammation process will stop and there will be a damaged axon remyelination. Astroglia then forms scarring of dead cells. Damage to axons, thus, is considered as a major cause of permanent neurological deficits.

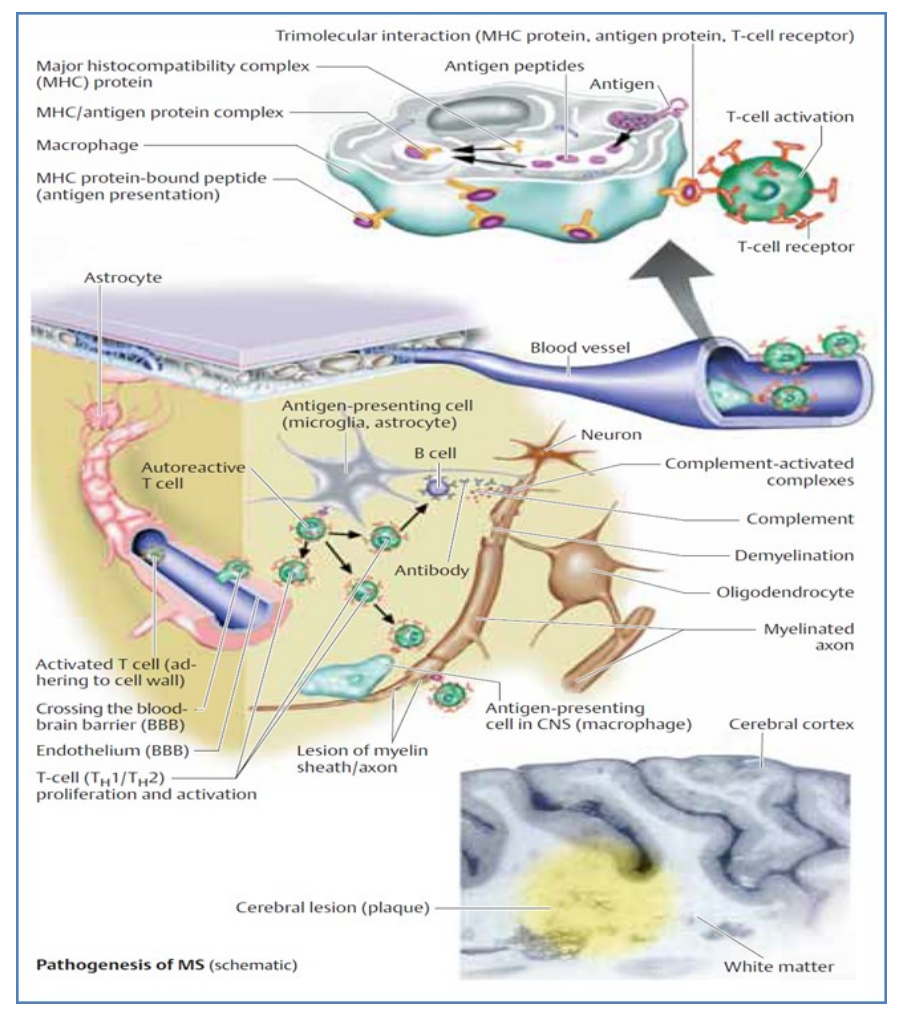

Figure 1. Schematical pathogenesis of multiple sclerosis ${ }^{11}$

\section{CLINICAL MANIFESTATIONS}

The symptoms and signs of MS vary widely because the disease can involve the central nervous system anywhere. The most common symptoms and signs are disturbance or loss of sensation due to interference with the spinothalamic tract or posterior part of the spinal cord, visual disturbance due to optic neuritis, paralysis or spasticity due to disturbances in the corticospinal tract, tremor and coordination disorders due to disturbances of the cerebellum or spinocerebelar pathway, as well as abnormalities in cranial nerve function due to brain stem disturbances. Besides, gastrointestinal disorders, urinary tract and sexual dysfunction occur in two urinary tract and sexual dysfunction occur in two thirds of patients due to spinal cord disturbance. Tiredness, depression and cognitive impairment are also considered as common symptoms. ${ }^{1,11}$

The typical signs of MS, moreover, are the clinical symptoms and the varied course of the disease. Multiple sclerosis can be divided into four clinical categories, namely Clinically Isolated Syndrome (CIS), Relapsing Remitting Multiple Sclerosis (RRMS), Secondary Progressive Multiple Sclerosis (SPMS) and Primary Progressive Multiple Sclerosis (PPMS). The onset of relapse and remission is found in $85-90 \%$ of patients. Approximately $40-50 \%$ of RRMS patients stop getting attacks and progress to progressive neurodegenerative disease due to chronic CNS inflammation, known as SPMS. SPMS patients are those who do not have the onset of acute attacks, but from the beginning there is a gradual clinical deterioration. ${ }^{1,12}$

\section{LABORATORY DIAGNOSIS}

The diagnosis of multiple sclerosis is confirmed using the 2010 revised McDonald's criteria based on clinical manifestations and multiple investigations, including Magnetic Resonance Imaging (MRI), Cerebrospinal Fluid (CSF) screening and evoked potential (visual, brainstem and somatosensory) examinations. ${ }^{13-}$ 15

\section{Cerebrospinal Fluid Inspection}

1. Cerebrospinal fluid analysis

Cerebrospinal fluid of MS patients is generally colorless and crystal clear. Normal glucose levels, normal or slightly increased total protein and normal cell count are also found although sometimes there is an increase in mononuclear cell dominance. A total protein count is more than $100 \mathrm{mg} / \mathrm{dL}$ and an increase in the number of cells up to 100 cells $/ \mathrm{mm} 3$ is very rare in MS patients, but if found should be considered as other diagnoses. ${ }^{2,16,17}$

\section{IgG Index}

IgG index or "Link Index" is the ratio of CSS IgG to CSS albumin compared with the ratio of serum IgG to serum albumin. Increased IgG index in MS patients ( $>0.7)$, therefore, can indicate the presence of intrathecal IgG synthesis. The increased IgG index can be found in $80 \%$ of MS patients. Examination of the IgG index together with Oligoclonal Immunoglobulin bands, furthermore, can increase its sensitivity to $90 \%$. The increased IgG index, nevertheless, is also obtained in other several diseases, namely neurosyphilis, acute inflammatory polyradiculoneu- 
ropathy and subacute sclerosing panWencephalitis. ${ }^{17,18}$

3. Oligoclonal Immunoglobulin bands (OCBs)

The discovery of OCB suggests immunoglobulin synthesis and intrathecal inflammatory processes. It is found in over $90 \%$ of patients and also considered as one of the laboratory criteria that supports the diagnosis of MS. The sensitivity of this test is $90 \%$ with the specificity of $95 \% .{ }^{2,16,19}$

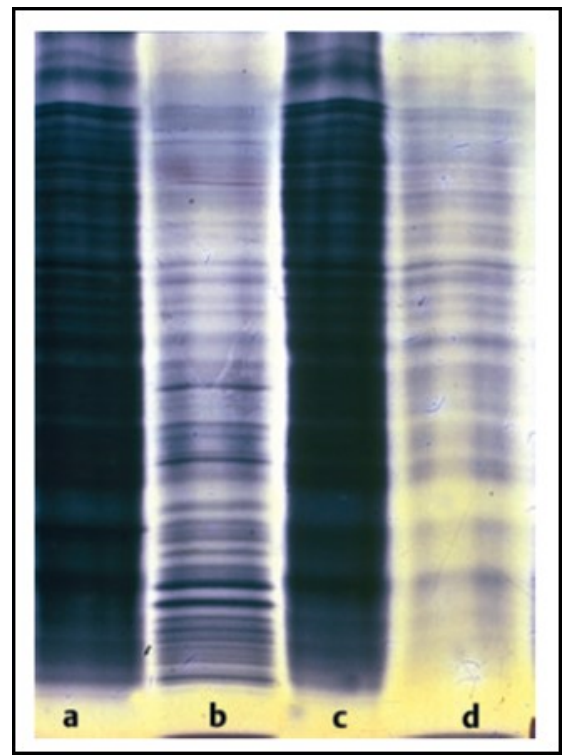

Figure 2. Oligoclonal bands in serum (a) and CSS (b) of MS patients; compared with serum (c) and CSS (d) of normal individuals. ${ }^{4}$

The most widely used method of IgG index examination is Isoelectric Focusing (IEF). This method uses a $\mathrm{pH}$ gradient to separate IgG populations which are then transferred into nitrocellulose or other membranes. This examination should be performed simultaneously with serum samples to compare the results. Next, serum electrophoresis and CSS together with IEF results then are read with IgG immunoblot to see the presence of OCB in CSS and the absence of OCB in serum. When electrophoresis examination is performed on the normal cerebrospinal fluid (CSS), it will generate results in a homogeneous blur of immunoglobulin, whereas in MS patients there will be some lines showing excessive antibody production (Figure 2). ${ }^{1,16,19}$

\section{PROGNOSIS}

Life expectancy in MS patients is only slightly reduced and survival is related to the disability occurring. A total of $30 \%$ of untreated patients will get physical disability within 20-25 years after the on- set. Good prognostic indicators in MS patients are age less than 40 years, no disruption to the cerebellum, RRMS group, short duration of relapse and normal walking ability. The benign case is defined as a low recurrence frequency and shows only mild defects within the first 15 years. The disease is called malignant if it shows severe disability within the first 5 years. However, one-third of patients do not show severe disability in the first 10 years. ${ }^{4,11,21}$

\section{REFERENCES}

1. Brass $\mathrm{S}$, Weiner $\mathrm{H}$, Hafler D. Multiple Sclerosis. In: Rose $\mathrm{N}$ and Mackay I. The Autoimmune Disease. USA, Elsevier, 2006; 615-27.

2. Milo $R$ and Miller A. Multiple Sclerosis. In: Shoenfeld $Y$, Cervera R, Gershwin M. Diagnostic Criteria in Autoimmune Disease. USA, Humana Press, 2008; 401-6.

3. Tsokos G and Virella G. Organ Spesific Autoimmune Disease. In: Virella G. Medical Immunology. $6^{\text {th }}$ Ed., USA, Informa, 2007; 255-7.

4. Mumenthaler $\mathrm{M}$, Mattle $\mathrm{H}$ and Taub $\mathrm{E}$. Fundamentals of Neurology. New York, Thieme, 2006; 156-60.

5. Chamberlin S and Narins B. The Gale Encyclopedia of Neurological Disorders. USA, Thomson Gale, 2005; 561-5.

6. Chapel H, Haeney M, Misbah S and Snowden N. Essential of Clinical Immunology. $6^{\text {th }}$ Ed., UK, Wiley Blackwell, 2014; 314-8.

7. Pithadia A, Jain S and Navale A. Pathogenesis and Treatment of Multiple Sclerosis (MS). The Internet Journal of Neurology, 2008; 10(2): 1-13.

8. Matilde Inglese. Multiple Sclerosis: New Insights and Trends. Am J Neuroradiol, 2006; 27: 954-57.

9. Rejdak K, Jackson S and Giovannoni G. Multiple Sclerosis: a Practical Overview for Clinicians. British Medical Bulletin, 2010; 95: 79-104.

10. Hoppenbrouwers I and Hintzen R. Genetics of Multiple Sclerosis. Biochymica et Biophysica Acta, 2011; 1812: 194 201.

11. Rohkamm R. Color Atlas of Neurology. New York, Thieme, 2004; 214-21.

12. Loma I and Heymann R. Multiple Sclerosis: Pathogenesis and Treatment. Current Neuropharmacology, 2011; 9: 40916.

13. Palace J. Making the Diagnosis of Multiple Sclerosis. J neurol Neurosurg Psychiatry, 2001; 71(II): ii3-ii8.

14. Calabresi P. Diagnosis and Management of Multiple Sclerosis. American Academy of Family Physician, 2004; 70(10): 1935-43.

15. Polman C, Reingold S, Banwell B, Clanet M, Cohen J, et al. Diagnostic Criteria of Multiple Sclerosis: 2010 Revisions to the McDonald Criteria. American Neurological Association, 2011; 69: 292-302. 
16. Deisenhammer F, Bartos A, Egg R, Gilhus N, Giovannoni $\mathrm{G}$, et al. Routine Cerebrospinal Fluid (CSF) Analysis. In Gilhus N, Barnes M and Brainin M. European Handbook of Neurological Management: Volume 1. $2^{\text {nd }}$ Ed., New York, Blackwell Publishing, 2011; 5-14.Kottill WR. Cerebrospinal Fluid in Multiple Sclerosis. Annals of Indian Academy of Neurology, 2009; 12(4): 246-53.

17. Kottill WR. Cerebrospinal Fluid in Multiple Sclerosis. Annals of Indian Academy of Neurology, 2009; 12(4): 246-53

18. Mayo Clinic Medcal Laboratories. Cerebrospinal Fluid (CSF) Indes IgG. Available from: http:// www.mayomedicallaboratories.com/test-catalog. Accessed on December $17^{\text {th }} 2016$.
19. Mayo Clinic Medcal Laboratories. Oligoclonal Banding, Serum and Spinal Fluid. Available at: http:// www.mayomedicallaboratories.com/test-catalog. Accessed on: December $17^{\text {th }} 2016$.

20. Bloch S, Callin S, Coggrave M, Smith A, Dods V, et al. Multiple Sclerosis Information for Health and Social Care Professional. Available from: www. mstrust.org.uk. Accessed on: May $28^{\text {th }} 2016$.

21. Luzzio C. Multiple Sclerosis. Available from: www. emedicine.medscape.com/article/980685. Last Update: Jan 2016. Accessed on: May $28^{\text {th }} 2016$ 